PROCEEDINGS OF THE

AMERICAN MATHEMATICAL SOCIETY

Volume 134, Number 10, October 2006, Pages 2943-2950

S 0002-9939(06)08333-X

Article electronically published on April 11, 2006

\title{
BEURLING-NEVANLINNA INEQUALITY FOR SUBFUNCTIONS OF THE STATIONARY SCHRÖDINGER OPERATOR
}

\author{
ALEXANDER KHEYFITS \\ (Communicated by Juha M. Heinonen) \\ To Iossif V. Ostrovskii on the occasion of his 70th Anniversary
}

\begin{abstract}
The classical Beurling-Nevanlinna upper bound for subharmonic functions is extended to subsolutions of the stationary Schrödinger equation.
\end{abstract}

Let $u$ be a subharmonic function in the disk $D_{R}=\{|z|<R\}$ in the complex plane. By the maximum principle, if

$$
\limsup _{z \rightarrow \partial D_{R}} u(z) \leq M
$$

then $u \leq M$ in the whole $D_{R}$, and since a constant function $u(z) \equiv M$ is subharmonic, (1) cannot be improved. However, if some additional information is available, the latter inequality can be made more precise. Thus, improving on the preceding results of H. Milloux and E. Schmidt, A. Beurling [2] and R. Nevanlinna [10] proved that if in addition to (1)

$$
\inf _{|z|=r} u(z) \leq m, \forall r \in[0, R),
$$

then everywhere in $D_{R}$

$$
u(z) \leq \delta_{0}(r) m+\left(1-\delta_{0}(r)\right) M,
$$

where

$$
\delta_{0}(r)=\frac{2}{\pi} \arcsin \frac{R-r}{R+r} .
$$

Here we follow L. Hörmander [6, p. 194]; Beurling and Nevanlinna actually proved a more general result, assuming that $(2)$ is known only on a subset of $[0, R)$. For the relevant references see W. Hayman [5, p. 289].

Our goal is to extend (3) to weak solutions of the stationary Schrödinger inequality $-L_{c} u \equiv \Delta u-c(z) u \geq 0$ in the disk $D_{R}, \Delta$ being the Laplace operator. We call these functions subfunctions or $c$-subfunctions, to be more precise. Solutions of the equation $\Delta u-c(z) u=0$ are called $c$-harmonic functions. One can find the necessary properties of subfunctions in [8, 9] and the references therein.

We always assume that the potential $c(z)$ is a nonnegative function in the disk $D_{R}$ and $c(z) \in L^{2}\left(D_{R-\epsilon}\right)$ for every $\epsilon, 0<\epsilon<R$. This assumption is sufficient

Received by the editors May 19, 2004 and, in revised form, April 26, 2005.

2000 Mathematics Subject Classification. Primary 31A05, 30C80, 35J10.

Key words and phrases. Beurling-Nevanlinna inequality, subharmonic functions associated with the stationary Schrödinger operator. 
to derive the local properties of subfunctions. However, for our purposes we must control the behavior of the potential near the boundary. Thus, we assume that $c(z)$ has an appropriate radial majorant, that is, there exist a function $Q(r), 0 \leq r<R$, and a number $\varepsilon>0$ such that

$$
0 \leq c(z) \leq Q(|z|) \in L^{2+\varepsilon}(0, R), \forall z \in D_{R} .
$$

The results are stated in terms of special solutions of the following ordinary differential equations:

$$
f^{\prime \prime}(r)+\frac{1}{r} f^{\prime}(r)-Q(r) f(r)=0,0<r<R,
$$

and

$$
f^{\prime \prime}(r)+\frac{1}{r} f^{\prime}(r)-\left(\frac{(2 l+1)^{2}}{4 r^{2}}+Q(r)\right) f(r)=0, l=0,1,2, \ldots, 0<r<R .
$$

Under our assumptions each of these equations has a fundamental system of positive solutions, denote them $\left\{V_{0}, U_{0}\right\}$ for (4) and $\left\{V_{2 l+1}, U_{2 l+1}\right\}$ for (5), such that $U_{0}\left(0^{+}\right)=U_{2 l+1}\left(0^{+}\right)=+\infty, U_{0}\left(R^{-}\right)=U_{2 l+1}\left(R^{-}\right)=0, V_{2 l+1}\left(0^{+}\right)=0$ for all $l \geq 0$ and $V_{0}\left(0^{+}\right) \geq 0$ (see, e.g., 4, Chap. 11]). The functions $U_{0}, U_{2 l+1}$ monotonically decay, while $V_{2 l+1}$ and $V_{0}$ monotonically increase (the latter may be constant) and are bounded when $r \rightarrow R$, that is, $V_{0}\left(R^{-}\right)<\infty$ and $V_{2 l+1}\left(R^{-}\right)<\infty$. Now we can state our result. As always, $a^{+}=\max \{a ; 0\}$.

Theorem 1. Let a potential $c(z), z \in D_{R}$, satisfy our assumptions and let a c-subfunction $u$ satisfy (1) and (2). Then

$$
u(z) \leq \delta(r) m+(1-\delta(r)) M^{+},
$$

where

$$
\delta(r)=\frac{V_{0}(r)}{V_{0}(R)}-\frac{4}{\pi} \sum_{l=0}^{\infty} \frac{(-1)^{l} V_{2 l+1}(r)}{(2 l+1) V_{2 l+1}(R)} .
$$

The statement is precise in the sense that $M^{+}$in (6) cannot be replaced by $M$.

Moreover, inequality (3) in general fails for $c-$ subfunctions unless $c=0$ almost everywhere.

Remark 1. The appearance of $M^{+}$in (6) is due to the fact that for the operators under consideration the maximum principle has a restricted validity - only a positive maximum cannot be attained inside the domain.

Proof. Since $(\Delta-Q)\left(u-M^{+}\right)=(\Delta-c) u+(c-Q)\left(u-M^{+}\right)+c M^{+} \geq 0$, perhaps in the sense of distributions, $u(z)-M^{+}$is a nonpositive $Q$-subfunction. By the Riesz-Herglotz integral representation [7,

$$
u(z)-M^{+}=-\int_{D_{R}} G(z, \zeta) d \mu(\zeta)-\int_{S_{R}} \frac{\partial G(z, \zeta)}{\partial n(\zeta)} d \sigma(\zeta),
$$

where $S_{R}=\partial D_{R}, d \mu$ and $d \sigma$ are, respectively, the Riesz measure and the boundary measure of $u, G$ is Green's function of the operator

$$
L_{Q}=-\Delta+Q(|z|) I
$$

in $D_{R}$ with the Dirichlet boundary conditions, and $\partial G / \partial n$ is its derivative with respect to the inward normal to $S_{R}$. It is known (see, for instance, 9] and the references therein) that $G$ exists, $G(z, \zeta)>0$ and $\partial G / \partial n \geq 0$. 
We follow the argument of [6, pp. 194-196]. However, unlike the case $c=0$, we do not have an explicit integral representation of Green's function $G$, so the extension is not straightforward and we cannot expect in general to derive an explicit formula for the function $\delta(r)$.

Project all associated masses of $u$ on the positive real axis along concentric circumferences. Define a measure $d \mu^{*}$ on $[0, R)$ such that

$$
\int \varphi(r) d \mu^{*}(r)=\int \varphi(|z|) d \mu(z)
$$

for any function $\varphi \in C_{0}([0, R))$, denote $\sigma^{*}=\int d \sigma \geq 0$, and consider a function

$$
v(z)=-\int_{[0 . R)} G(z, r) d \mu^{*}(r)-\left.\sigma^{*} \frac{\partial G(z, \zeta)}{\partial n(\zeta)}\right|_{\zeta=R} .
$$

In the proof we need some inequalities between the functions $u$ and $v$. To establish them, we estimate changes of Green's function $G$ when $z$ moves along circumferences $|z|=r, 0<r<R$. To this end, the following definition [1] is helpful.

$A$ function $b(r, \theta)$ is said to be symmetric on an annulus

$$
A\left(r_{1}, r_{2}\right)=\left\{(r, \theta) \mid r_{1}<r<r_{2}, 0 \leq \theta \leq 2 \pi\right\}
$$

if $b(r, \theta)=b(r, 2 \pi-\theta), \forall r, r_{1}<r<r_{2}$.

Lemma 1. If $\rho$ is real, then $G\left(r e^{i \theta}, \rho\right)$ is symmetric on $D_{R}$. Next, for $0<\theta<\pi$,

$$
\frac{\partial}{\partial \theta} G\left(r e^{i \theta}, \rho\right) \leq 0
$$

and so $\frac{\partial}{\partial \theta} G\left(r e^{i \theta}, \rho\right) \geq 0$ for $\pi<\theta<2 \pi$.

Proof. The symmetry of $G$ follows immediately, since $Q(r)$ is a radial potential. The formula

$$
(\Delta-Q(r))\left(\frac{\partial b}{\partial \theta}\right)=\frac{\partial}{\partial \theta}(\Delta b-Q(r) b)
$$

is readily verified for smooth functions $b(r, \theta)$ on the semi-annulus

$$
A^{+}\left(r_{1}, r_{2}\right)=\left\{(r, \theta) \mid 0 \leq r_{1}<r<r_{2}, 0<\theta<\pi\right\} .
$$

Thus, since $G(z, \zeta)$ is $Q$-harmonic for $z \neq \zeta$, the function $b$ defined by

$$
b(r, \theta)=\frac{\partial}{\partial \theta} G((r, \theta), \rho)
$$

is also $Q$-harmonic in the punctured disk $D_{R} \backslash\{(\rho, 0)\}$.

Moreover, $b(r, \theta) \rightarrow 0$ as $(r, \theta) \rightarrow(R, \theta)$, because $Q(r) \in L^{2+\varepsilon}(0, R)$ and all points of $S_{R}$ are regular for both $L_{c}$ and $L_{Q}$. Also $\lim b(r, \theta)=0$ as $\theta \rightarrow 0$ and $\theta \rightarrow \pi, r \neq \rho$, by virtue of the symmetry of $G$. In addition, $\lim _{\sup _{z \rightarrow \rho}} b(z) \leq 0$.

Indeed, the equation $-\Delta u+Q(|z|) u(z)=f(z)$ can be rewritten in a standard way as an integral equation

$$
u(z)=\int_{D_{R}} g(z, \zeta) f(\zeta) d \zeta+\int_{D_{R}} g(z, \zeta) u(\zeta) d \zeta,
$$

where $g$ is Green's function of the Laplace operator $-\Delta$ in the disk $D_{R}$ with zero boundary values. Iterating (8), we can derive Green's function $G$ of the operator $L_{Q}$ and study its properties; see [9] for details. In particular, the following 
representation holds true:

$$
G(z, \rho)=\frac{1}{2 \pi} \ln \frac{1}{|z-\rho|}+a \int_{D_{R}} \ln \frac{1}{|z-t|} Q(|t|) \ln \frac{1}{|t-\rho|} d t+h(z, \rho),
$$

where $a$ is a constant and $h$ is a function with bounded first partial derivatives, perhaps in the sense of distributions. Now, since $Q \in L^{2+\varepsilon}$, we easily conclude that the integral in (9) also has bounded first derivatives.

Thus, $b(r, \theta)<\epsilon, \epsilon>0$, in some small vicinity of the point $(\rho, 0)$, and also $b$ is $Q$-harmonic outside this vicinity. By the maximum principle, $\frac{\partial G}{\partial \theta}=b(z) \leq 0$ in the semi-annulus $A^{+}$.

Corollary 1. Lemma 1 implies immediately that

$$
G(-|z|, \rho) \leq G(z, \rho) \leq G(|z|, \rho) .
$$

To handle the second term, $\partial G / \partial n$, in the right-hand side of (7), we note that since Green's function vanishes at regular boundary points and is symmetric, then (10) with $\rho \rightarrow R$ implies

$$
P(-|z|, R) \leq P\left(z, R e^{i \theta}\right) \leq P(|z|, R),
$$

where $P\left(z, R e^{i \theta}\right)=\left.\frac{\partial}{\partial n} G(z, \zeta)\right|_{\zeta=R e^{i \theta}}$ is the $Q$-harmonic Poisson kernel for the disk $D_{R}$. Thus,

$$
u(z)-M^{+} \leq v(-r), r=|z|,
$$

for $|z|<R$, where the function $v$ is given by (7). Moreover,

$$
\begin{gathered}
v(r)=-\int_{[0, R)} G(r, \rho) d \mu^{*}(\rho)-\sigma^{*} P(r, R) \\
\leq-\int_{[0, R)} G(z, \rho) d \mu^{*}(\rho)-\sigma^{*} P\left(z, R e^{i \theta}\right)=u(z)-M^{+},
\end{gathered}
$$

hence

$$
v(r) \leq \inf _{|z|=r} u(z)-M^{+} \leq m-M^{+}, 0 \leq r<R
$$

To find an analog of the function $\delta_{0}$ in (3), we first solve the following Dirichlet problem in the slit disk $D_{R}^{-}=D_{R} \backslash\{0 \leq r<R\}$ :

$$
\begin{cases}L_{Q} w(z)=0 & \text { for } z \in D_{R}^{-}, \\ w\left(R e^{i \theta}\right)=1 & \text { if } \theta \neq 0, \\ w\left(r, 0^{+}\right)=w\left(r, 2 \pi^{-}\right)=0 & \text { if } 0<r<R .\end{cases}
$$

Separating variables by substituting $w(r, \theta)=f(r) \Theta(\theta)$, we deduce for the angular component the equation $\Theta^{\prime \prime}(\theta)+\lambda \Theta(\theta)=0,0<\theta<2 \pi$, so $\Theta_{\lambda}(\theta)=A_{\lambda} \sin (\sqrt{\lambda} \theta)+$ $B_{\lambda} \cos (\sqrt{\lambda} \theta)$. Due to the nonnegativity of the potential $Q$, the spectrum of the operator $L_{Q}$ is nonnegative, thus $\lambda \geq 0$.

An equation for the radial component of $w$ is (cf. (5))

$$
f^{\prime \prime}+\frac{1}{r} f^{\prime}-\left(\frac{\lambda}{r^{2}}+Q(r)\right) f(r)=0,0<r<R .
$$

It is known (see, e.g., 4, Chap. 11]) that (12) has a fundamental system of positive solutions, $V_{\lambda}(r)$ and $U_{\lambda}(r)$, such that $U_{\lambda}\left(0^{+}\right)=\infty, U_{\lambda}(r)$ monotonically decreases to zero as $0<r \rightarrow R$, while $V_{\lambda}\left(0^{+}\right) \geq 0$, and $V_{\lambda}(r)$ does not decrease as $0<r \rightarrow R$. 
Moreover, if $\lambda>0$ or $Q(r)$ is positive on a set of positive measure, then $V_{\lambda}\left(0^{+}\right)=0$ and $V_{\lambda}(r)$ monotonically increases as $0<r \rightarrow R$. Thus, the general solution of (12) is

$$
f_{\lambda}(r)=C_{\lambda} V_{\lambda}(r)+D_{\lambda} U_{\lambda}(r) .
$$

In the proof we do not need the general solution of the Dirichlet problem (11), so we fix $D_{\lambda}=0$ and $C_{\lambda}=1$, and consider a radial solution $f_{\lambda}(r)=V_{\lambda}(r)$, leading to a solution of (11),

$$
w_{\lambda}(r, \theta)=\left\{A_{\lambda} \sin (\sqrt{\lambda} \theta)+B_{\lambda} \cos (\sqrt{\lambda} \theta)\right\} V_{\lambda}(r) .
$$

Considering the boundary conditions at the slit, we get $B_{\lambda}=0$ since $V_{\lambda}(r) \neq 0$ for $r>0$, and $A_{\lambda} \sin (2 \pi \sqrt{\lambda})+B_{\lambda} \cos (2 \pi \sqrt{\lambda})=0$, implying $\sin (2 \pi \sqrt{\lambda})=0$. Thus, $\lambda=\lambda_{k}=\frac{k^{2}}{4}, k=0,1,2, \ldots$,

$$
w_{\lambda}(r, \theta)=w_{k}(r, \theta)=A_{k} V_{k}(r) \sin \left(\frac{k}{2} \theta\right), k=0,1,2, \ldots,
$$

and $w(r, \theta)=\sum_{k=0}^{\infty} A_{k} V_{k}(r) \sin \left(\frac{k}{2} \theta\right)$. Next, the boundary condition at $r=R$ gives

$$
w(R, \theta)=\sum_{k=0}^{\infty} A_{k} V_{k}(R) \sin \left(\frac{k}{2} \theta\right)=1,0<\theta<2 \pi
$$

From here,

$$
A_{k}= \begin{cases}0 & \text { if } k \text { is even } \\ \frac{4}{\pi k V_{k}(R)} & \text { if } k=2 l+1 \text { is odd }\end{cases}
$$

and we have constructed a solution of (11),

$$
w(z)=\frac{4}{\pi} \sum_{l=0}^{\infty} \frac{V_{2 l+1}(r)}{(2 l+1) V_{2 l+1}(R)} \sin \left(\left(l+\frac{1}{2}\right) \theta\right),
$$

which we sought.

Since constants are not $c$-harmonic functions (unless $c \equiv 0$ ), to get a solution of (11) with zero boundary values at $S_{R}$, we consider a radial positive $Q$-harmonic function $w_{1}(z)=\frac{V_{0}(r)}{V_{0}(R)}, 0 \leq r=|z| \leq R$, in the slit disk $D_{R}^{-}$, where $V_{0}$ is a growing solution of equation (4). It is obvious that $w_{1}(R)=1,0 \leq \theta \leq 2 \pi$, while $w_{1}(0)=V_{0}(0) / V_{0}(R) \geq 0$, and $0<w_{1}(r)<1$ for $0<r<R$. By the maximum principle, $w(z) \leq w_{1}(z)$ in $\overline{D_{R}^{-}}$, so that the function

$$
W(z)=w(z)-w_{1}(z)=\frac{4}{\pi} \sum_{l=0}^{\infty} \frac{V_{2 l+1}(r)}{(2 l+1) V_{2 l+1}(R)} \sin \left(\left(l+\frac{1}{2}\right) \theta\right)-\frac{V_{0}(r)}{V_{0}(R)}
$$

is a negative $Q$-harmonic function in $D_{R}^{-}, W(R, \theta)=0$ for $0<\theta<2 \pi$, and $W\left(r, 0^{+}\right)=W\left(r, 2 \pi^{-}\right)=-\frac{V_{0}(r)}{V_{0}(R)}>-1$ for $0<r<R$.

As we have proved, the function $v$, defined by (7), satisfies $v(z) \leq 0$ in $D_{R}$, and $v(r) /\left(M^{+}-m\right) \leq-1,0<r<R$. By the maximum principle,

$$
\frac{v(z)}{M^{+}-m} \leq W(z), z \in \overline{D_{R}^{-}}
$$


hence

$$
\begin{gathered}
u(z)-M^{+} \leq v(-r) \leq\left(M^{+}-m\right) W(-r) \\
=\left(M^{+}-m\right)\left\{\frac{4}{\pi} \sum_{l=0}^{\infty} \frac{(-1)^{l} V_{2 l+1}(r)}{(2 l+1) V_{2 l+1}(R)}-\frac{V_{0}(r)}{V_{0}(R)}\right\}
\end{gathered}
$$

or

$$
u(z) \leq \delta(r) m+(1-\delta(r)) M^{+},
$$

where

$$
\delta(r)=-W(r, \pi)=\frac{V_{0}(r)}{V_{0}(R)}-\frac{4}{\pi} \sum_{l=0}^{\infty} \frac{(-1)^{l} V_{2 l+1}(r)}{(2 l+1) V_{2 l+1}(R)}>0 .
$$

The latter series converges by the Abel test [3, p. 307], since $\left\{\frac{V_{2 l+1}(r)}{V_{2 l+1}(R)}\right\}$ is monotone in $l$ for any $r, 0 \leq r<R$, 4, Chap. 11, Sect. 6, Cor. 6.5].

Now consider a negative subfunction $-w_{1}(z)$. It satisfies (1)-(2) with $M=$ $0, m=-1$, and if it were possible to replace $M^{+}$in (6) with $M$, we would have $-\frac{V_{0}(r)}{V_{0}(R)} \leq-1,0 \leq r<R$, contrary to the definition of $V_{0}$.

Moreover, if we could substitute a simpler weight $\delta_{0}$ (see (3)) in (6) instead of the general weight $\delta$, we would get from (6) for the same function $-w_{1}(z)$,

$$
\frac{2}{\pi} \arcsin \frac{R-r}{R+r} \leq \frac{V_{0}(r)}{V_{0}(R)}
$$

an obviously contradictory inequality as $r \rightarrow 0^{-}$, unless $V_{0}$ is constant, which holds only in the classical case $c=0$. The proof of Theorem 1 is complete.

Corollary 2. Under the conditions of Theorem 1,

$$
\begin{gathered}
\sup _{0 \leq r \leq R} \inf _{|z|=r} u(z) \geq M^{+}(R)-\frac{M^{+}(R)-M(r)}{\delta(r)} \\
\geq M(r)-\inf _{0 \leq r<R} \frac{M^{+}(R)-M(r)}{\delta(r)} .
\end{gathered}
$$

Proof. Since $u(z) \leq \delta(r) m+(1-\delta(r)) M^{+}, r=|z|$, we have $M(r)=M_{u}(r) \leq$ $\delta(r) m+(1-\delta(r)) M^{+}$. Thus

$$
m \geq \frac{M(r)-(1-\delta(r)) M^{+}(R)}{\delta(r)}=M^{+}(R)-\frac{M^{+}(R)-M(r)}{\delta(r)},
$$

and the conclusion follows.

Corollary 3. Let $u$ and $v$ be c-subfunctions in $D_{R}$ and $M(R)=M_{u}(R)<\infty$. Then

$$
v(0) \leq \sup _{|z|<R}(u(z)+v(z))-M^{+}(R)+\inf _{0 \leq r \leq R} \frac{M^{+}(R)-M(r)}{\delta(r)} .
$$

Proof. Indeed,

$$
v(0) \leq \sup _{|z| \leq r} v(z) \leq \sup _{|z| \leq r}(u+v)-\inf _{|z|=r} u \leq \sup _{|z|<R}(u(z)+v(z))-\inf _{|z|=r} u(z),
$$

and it suffices now to apply Corollary 2 . 
We consider two examples. First, let $Q(r)=q r^{-2}$, where $q$ is a positive constant. In this case, $V_{0}(r)=A_{0} r^{\sqrt{q}}, V_{2 l+1}(r)=A_{l} r^{\sqrt{(l+1 / 2)^{2}+q}}$, and

$$
\delta(r)=\left(\frac{r}{R}\right)^{\sqrt{q}}-\frac{4}{\pi} \sum_{l=0}^{\infty} \frac{(-1)^{l}}{2 l+1}\left(\frac{r}{R}\right)^{\sqrt{(l+1 / 2)^{2}+q}} .
$$

It should be mentioned that $\delta(R)=\delta_{0}(R)=0$. However, $\delta(0)=0 \neq \delta_{0}(0)=1$, unless the potential vanishes almost everywhere on $(0, R)$; in the latter case $\delta(0)=$ $\delta_{0}(0)=1$.

As the second example, we consider a constant potential $c(z) \equiv Q(r)=q \geq 0$. Now equation (12) reduces to the Bessel differential equation, $V_{0}(r)=A J_{0}(i \sqrt{q} r)$, $V_{2 l+1}(r)=A_{l} J_{l+1 / 2}(i \sqrt{q} r)$, where $J_{\nu}$ is a Bessel function of the first kind, and

$$
\delta(r)=\frac{J_{0}(i \sqrt{q} r)}{J_{0}(i \sqrt{q} R)}-\frac{4}{\pi} \sum_{l=0}^{\infty} \frac{(-1)^{l} J_{l+1 / 2}(i \sqrt{q} r)}{(2 l+1) J_{l+1 / 2}(i \sqrt{q} R)} .
$$

In particular, if $q=0$, then $V_{2 l+1}(r)=A_{l} r^{l+1 / 2}$,

$$
w(z)=\frac{4}{\pi} \sum_{l=0}^{\infty} \frac{(r / R)^{2 l+1}}{l+1 / 2} \sin ((l+1 / 2) \theta)=\frac{2}{\pi} \arctan \frac{2 \sqrt{r / R} \sin \frac{\theta}{2}}{1-r / R}
$$

So

$$
w(r, \pi)=\frac{4}{\pi} \sum_{l=0}^{\infty} \frac{(-1)^{l}(r / R)^{l+1 / 2}}{2 l+1}=\frac{2}{\pi} \arctan \frac{2 \sqrt{r / R}}{1-r / R} .
$$

In this case we can set $w_{1}(z)=1$, so

$$
\delta(r)=\delta_{0}(r)=1-\frac{2}{\pi} \arctan \frac{2 \sqrt{r / R}}{1-r / R}=\frac{2}{\pi} \arcsin \frac{R-r}{R+r},
$$

and we arrive at the classical inequality (3) [6, p. 194].

\section{ACKnowledgement}

The author is grateful to Professor L. Hörmander and to a referee for useful advice. This work was partially supported by a grant from the City University of New York.

\section{REFERENCES}

[1] Baernstein II, A. and Taylor, B. A., Spherical rearrangements, subharmonic functions, and *-functions in n-space. Duke Math. J. 43(1976), 245-268. MR0402083 (53:5906)

[2] Beurling, A., Études sur un problème de majoration. Thèse, Uppsala, 1933.

[3] Bonic, R. A., Hajian, G. V., Cranford, E., and Krantz, S., Freshman Calculus. D. C. Heath and Co. Lexington, MA, 1971.

[4] Hartman, P., Ordinary Differential Equations. John Wiley \& Sons, New York - London Sydney, 1964. MR0171038 (30:1270)

[5] Hayman, W. K., Subharmonic Functions. Vol. 2. Academic Press, London - San Diego - New York - Berkeley - Boston - Sydney - Tokyo - Toronto, 1989. MR.1049148 (91f:31001)

[6] Hörmander, L. Notions of Convexity. Birkhäuser, Boston - Basel - Berlin, 1994. MR:1301332 (95k:00002)

[7] Kheyfits, A., The Riesz-Herglotz formula for generalized harmonic functions and their boundary behavior. Soviet Math. Dokl. 44(1992), 688-691. MR.1153552

[8] Levin, B. Ya. and Kheyfits, A., Asymptotic behavior of subfunctions of the Schrödinger operator in an $n$-dimensional cone. Soviet Math. Dokl. 38(1989), 109-112. MR0968495 (91h:35099) 
[9] Levin, B. Ya. and Kheyfits, A., Asymptotic behavior of subfunctions of the stationary Schrödinger operator. Preprint, http://arXiv/abs/math/021132896, 2002, 96 pp.

[10] Nevanlinna, R., Über eine Minimumaufgabe in der Theorie der konformen Abbildung. Ges. Wiss. Göttingen, Math. Phys. Kl. 37(1933), 103-115.

Graduate School and Bronx Community College of The City University of New York, Bronx, New York 10453

E-mail address: akheyfits@gc.cuny.edu 